\title{
Fetoscopy and Risk of latrogenic Preterm Premature Rupture of Membranes: Not as High as It May Seem (in Experienced Hands)
}

\author{
Eduard Gratacós \\ Maternal-Fetal Medicine Department (ICGON), Hospital Clinic-IDIBAPS, University of Barcelona and Centro de \\ Investigación Biomédica en Red de Enfermedades Raras (CIBER-ER), Barcelona, Spain
}

In this issue of Fetal and Diagnostic Therapy, Beck et al. [1] publish an interesting systematic review on the factors influencing the risk of preterm premature rupture of membranes (PPROM) in fetoscopy. While this is a difficult task considering the scarcity of consistent information among published papers, the authors manage to extract some very interesting conclusions. The manuscript provides important messages, which add to the body of knowledge in the field of fetoscopy. However, these messages must be interpreted properly in order to be translated into clinical practice and patient counseling.

Firstly, the analysis confirms previously reported figures that fetoscopy is associated with a relatively high rate of PPROM, up to $30 \%$, defined as that occurring earlier than the 37 th week of gestation. However, the correct interpretation of these numbers is that, after all, fetoscopy is possibly not associated with a huge increase in the rate of PPROM. Indeed, we must remember that we are dealing with abnormal pregnancies. Twin monochorionic pregnancies are the clearest example of this. In a multicenter prospective study including over 200 monochorionic twin pregnancies, baseline risk of spontaneous PPROM in those not complicated by twin-to-twin transfusion syndrome was $25 \%$ [2]. In the case of shunting for urinary tract obstruction, it is possible that the increase is more important. However, fetal malformations are associated with an increased risk of PPROM and preterm delivery. While we are not aware of any sufficiently large clinical series reporting the perinatal outcome of fetuses with lower urinary tract obstruction managed expectantly, there is abundant information on other types of malformation. For instance, neural tube defects present rates of preterm delivery of up to $38 \%$ [3]. Along the same lines, congenital diaphragmatic hernia, which was not analyzed in the review by Beck et al. [1], has been reported to be associated with up to $29 \%$ of PPROM in pregnancies managed expectantly [4]. With these numbers in mind, the $40-47 \%[4,5]$ reported for cases managed with tracheal occlusion indeed suggests a clear impact of fetoscopy, but not as impressively high as it may seem without any baseline comparison.

A second important message of Beck et al.'s review [1] is that the diameter of the fetoscopic instrument is an independent predictor of the risk of PPROM. This is interesting information which had not been previously demonstrated. Again, however, the statement requires some qualification in the sense that the analysis included a wide range of diameters $(0.8-5 \mathrm{~mm})$. Thus, while it is certainly true that $1-\mathrm{mm}$ instruments are associated with a substantially lower risk of PPROM than those of $5 \mathrm{~mm}$, the impact of changes in fractions of mm could possibly be negligible. This is relevant because in clinical practice we may easily be tempted to reduce the outer diameter of the trocar. Indeed, going from 10 to 8 French may pro-

\section{KARGER}

Fax +41613061234 E-Mail karger@karger.ch www.karger.com
(C) 2012 S. Karger AG, Base

$1015-3837 / 12 / 0311-0010 \$ 38.00 / 0$

Accessible online at:

www.karger.com/fdt
Eduard Gratacós

Maternal-Fetal Medicine Department (ICGON), Hospital Clinic-IDIBAPS

University of Barcelona, Sabino de Arana 1

ES-08028 Barcelona (Spain)

Tel. +34 93227 9946, E-Mail egratacos@ clinic.ub.es 
duce a sense of reducing invasiveness, but we are actually reducing from 3.3 to $2.7 \mathrm{~mm}$. Is this relevant to the chances of having complications? Of course this does not mean that we should not aim at the lowest possible diameter, as long as this does not compromise the duration of the operation. This brings us to the last relevant comment about Beck et al.'s manuscript.

Unfortunately, the authors could not analyze the influence of the duration of the procedure on the risk of PPROM. This reflects the great variability in the information reported in the papers analyzed for the review. However, we miss one important part of the history and are left without any confirmation about the plausible effect of duration on the risk of PPROM complications. Since Beck conscientiously reviewed the available literature, we have little hope of getting this information in a reasonable time. Notwithstanding this, the duration of surgery should have a clear impact on the risk of complications. Aside from the obvious influence in the production of local inflammation and bleeding at the uterine wall entry site, there is a direct effect on membranes. In previous ex vivo studies using human membranes, Devlieger et al. [6] showed that the duration of the puncture was more important than the needle diameter in determining the final size of the defect created. This is logical considering that holding a needle is always associated with some subtle trembling and displacement, which over time progressively increases the tear induced in the frail chorioamniotic membranes. In real fetoscopy we must add the shearing movements between the uterus and the maternal abdominal wall. Thus, even if we do not have confirming evidence, it seems reasonable that we should aim at reducing the duration of fetoscopic operations to the minimum in order to have smaller membrane defects and less chorioamniotic separation. On the one hand, this points to the discussion about diameter that we left unfinished in the previous paragraph. On considering whether reducing the diameter of the instrument, think well whether this will affect the duration of your procedure. A smaller fetoscope means poorer visibility and this may result in a much longer procedure, particularly if there is difficult placental access or if amniotic fluid might be stained. On the other hand, it points to the recurrent discussion about the importance of experience for uncommon procedures such as fetoscopy. Experience influences an adequate planning and performance of the procedure, including selection of the right entry site and the type of scope, and avoids the many errors made in the past, which in the end results in much shorter and straightforward procedures. A handful of centers in the world have now a huge experience in fetoscopy. Experience allows us to reduce substantially the rate of complications and improve outcomes [7, 8]. Fetoscopic centers will continue expanding worldwide and this is highly desirable. However, on starting a fetoscopy program we must aim at offering patients the fetoscopy of today, not that of 15 years ago. There is no need to repeat the same errors that pioneers made. Therefore, no center should be offering fetoscopy without adequately training their staff for a sufficient length of time in other centers with proven experience. This, however, is the subject of another discussion.

\section{References}

1 Beck V, Lewi P, Gucciardo L, Devlieger R: Preterm prelabor rupture of membranes and fetal survival after minimally invasive fetal surgery: a systematic review of the literature. Fetal Diagn Ther 2012;31:1-9.

-2 Lewi L, Jani J, Blickstein I, Huber A, Gucciardo L, Van Mieghem T, Doné E, Boes AS, Hecher K, Gratacós E, Lewi P, Deprest J: The outcome of monochorionic diamniotic twin gestations in the era of invasive fetal therapy: a prospective cohort study. Am J Obstet Gynecol 2008; 199:514.

- 3 Hüsler MR, Danzer E, Johnson MP, Bebbington M, Sutton L, Adzick NS, Wilson RD: Prenatal diagnosis and postnatal outcome of fetal spinal defects without Arnold-Chiari II malformation. Prenat Diagn 2009;29:10501057.
-4 Ruano R, Duarte SA, Pimenta EJ, Takashi E, da Silva MM, Tannuri U, Zugaib M: Comparison between fetal endoscopic tracheal occlusion using a 1.0-mm fetoscope and prenatal expectant management in severe congenital diaphragmatic hernia. Fetal Diagn Ther 2011;29:64-70.

-5 Jani JC, Nicolaides KH, Gratacós E, Valencia CM, Doné E, Martinez JM, Gucciardo L, Cruz R, Deprest JA: Severe diaphragmatic hernia treated by fetal endoscopic tracheal occlusion. Ultrasound Obstet Gynecol 2009; 34:304-310.
-6 Devlieger R, Gratacos E, Ardon H, Vanstraelen S, Deprest J: Factors influencing the flow rate through a surgical defect in human fetal membranes. Prenat Diagn 2002;22:201205.

7 Hecher K, Diehl W, Zikulnig L, Vetter M, Hackelöer BJ: Endoscopic laser coagulation of placental anastomoses in 200 pregnancies with severe mid-trimester twin-to-twin transfusion syndrome. Eur J Obstet Gynecol Reprod Biol 2000;92:135-139.

8 Lewi L, Gratacos E, Ortibus E, Van Schoubroeck D, Carreras E, Higueras T, Perapoch J, Deprest J: Pregnancy and infant outcome of 80 consecutive cord coagulations in complicated monochorionic multiple pregnancies. Am J Obstet Gynecol 2006;194:782-789. 